\title{
An Intelligent Prediction System for Oil Spill Movement
}

\author{
Haojin Wang, Jan Wolter and Jungfu Tsao \\ Texas A\&M University \\ Dept. of Computer Science College Station, TX 77843 USA. \\ Telephone: 409-845-1489. Fax:409-847-8578. \\ email: wolter@cs.tamu.edu
}

\begin{abstract}
The goal of the research reported here is to build an intelligent system for the prediction of oil slick movement to facilitate the emergency management in case of a marine oil spill. In this paper, three main aspects of this system are discussed. First, an overview of the system architecture presents the major components of the system and the interconnection between them. Then the methods for acquiring diverse types of knowledge and for fusing them into a functional system is described. Finally, issues related to the implementation of this system are discussed.
\end{abstract}

\section{Keywords}

oil spill movement, prediction, knowledge acquisition, knowledge integration

\section{INTRODUCTION}

\section{$1.1 \quad$ Issues}

The serious environmental, economic and social consequences of oil spill have been demonstrated by recent major oil spills. Previous research in the fields such as oceanography, marine-physics and others have concentrated on developing various mathematical models for oil slick movement on the surface of ocean. However, making precise predictions may never be possible, since neither the current state of the world nor future developments in the conditions such as weather, current and temperature on which an oil spill model depends, can be reliably known. Thus, it is not very meaningful or it is even misleading to give decision makers a single projection of oil slick movement. Instead, we seek to produce a set of projections of the most likely scenarios for the oil slick with the likelihood attached to each of the projections. This will give decision makers a more realistic picture of the real world. To achieve this, we must make sure that our estimate of how conditions could develop takes into account all of the available knowledge and information. However, to complicate the issue, much of the observed data is available only in an imprecise form. For 
example, the wind directions are only given in approximate terms such as "east," "northwest," or "northeast" while we are required to predict the trajectory of oil slick movement as precise as possible. Domain expert's knowledge usually can only be described by vague terms.

In addition to the uncertainty and impreciseness associated with data and operating environment, the diversity in types of knowledge and information needs to be taken into consideration. For example, there are forecasts generated by other experts, such as weather forecasts. Then, there are historical records that may have been collected for the spill region over the years about weather and currents. Also, the user of the prediction system serves as a domain expert to give the judgement on the on-going situation. Correspondingly, different knowledge acquisition methods are required.

The system architecture we developed in this research stresses the integration of a knowledge base with various mathematical models that simulate various oil motions in the ocean. At the core of the system is a knowledge acquisition module. The module features different methods for acquiring diverse types of knowledge and the fusion of different types of knowledge to produce a realistic assessment of the oil slick movement. An object-oriented paradigm is used to implement the system and major issues related to the implementation are discussed.

\subsection{Related research}

Intensive research activities on oil spill modeling first occurred in the 1970s as a response to potential danger the marine oil spill may pose and as a result of people's increasing awareness of importance of environmental conservation. The initial research was limited to certain disciplines such as oceanography, industrial engineering and others (Anderson, 1974; Fay, 1971; Williams, 1974). There are some characteristics common to all these research. They take input data as given and treat the issue of predicting oil slick movement as a purely mathematical modeling problem. The oil spill model, which model the oil slick movement, usually consists of several distint submodels. Each of the submodel simulates a type of oil slick motion, such as dispersion, weathering, spreading or transport. Weathering refers to reduction in amount of oil due to the factors such as evaporation, emulsification, and biodegradation of oil. This is affected by the type of oil and weather conditions (Samuels, 1982; Venkatesh, 1988). Transport refers to the motion of an oil parcel from one location to another and is mainly determined by surface wind and current. Dispersion refers to the process by which waves spread the oil across the water surface or into the water column. Spreading is the process in which the oil layer thins out due to gravity and surface tension (Venkatesh, 1990).

In a related project we have addressed the problem of interpreting recent observations of the weather and the oil movement (Tsao, 1993; Tsao, 1994). This in effect uses an oil spill model to construct a scenario that fits the available observations and agrees with our model's understanding of the behavior of oil slicks. This model-based understanding of uncertain observational data of the oil slick provides the initial conditions from which predictions of future conditions are projected. Since the model-based interpretation of oil slick behavior generally provides finer spatial resolution than forecasts, and since there is significant continuity in most parameters, this provides a good starting point for our predictions. 


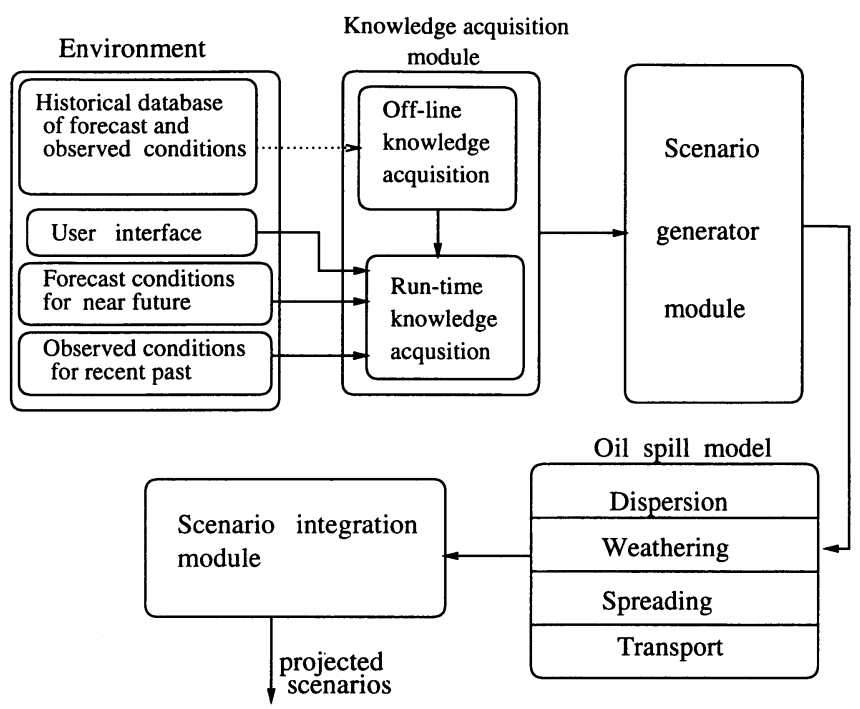

Figure 1 The Architecture for an Intelligent Prediction System

\section{SYSTEM ARCHITECTURE}

The intelligent prediction system consists of five distinct modules as depicted in Fig. 1. The functions each module performs and the connections between the modules are described in this section.

The first component of the system is the environment of the system which in turn consists of a user interface, a historical database of observed and forecast weather conditions, a set of forecast weather conditions for the near future and a set of observed conditions in the recent past. This environment module provides the sources from which the different types of knowledge are extracted during the learning stage as well as at run-time. The user interacts with the system through a user interface. There are two ways that a user can interact with the system. One is that the user enters the initial data values. For example, the user is prompted for the weather forecast, estimated volume of the initial spill, oil type, etc. The other way a user interacts with the system is that the user serves as a domain expert. The user is allowed to input the domain expertise into the system. One such expertise is his/her preference of one type of knowledge over another in deciding the most likely weather condition for the projection of the oil slick movement. The solid lines represent the run-time data/knowledge flow while the dotted lines the data flow during the learning stage.

The second component is the knowledge acquisition module. Its function is to build separate knowledge models based on the type of knowledge and the role each type of knowledge plays in generating a prediction and in inferring the likelihood of a given scenario. During the learning stage, the input to this module comes from the historical database of actual and forecast weather conditions. The run-time inputs come from users 
via a user interface and from the knowledge base created at the learning stage. The output of this module is a set of possible conditions. A detailed discussion on the knowledge acquisition is in the following section.

The third component of the system is the scenario generator module. It takes as inputs a set of possible conditions, makes the choice of the most likely condition and computes the likelihood measure for the condition. The major function of this module is to combine the results from the the knowledge acquisition module to satisfy two goals. One is to make a decision on the most likely condition from a set of possible conditions generated from the knowledge acquisition module for a given domain variable like wind or current. Then a set of the most likely conditions is input into the oil spill model to produce a projection of oil slick movement. The other goal is to combine the knowledge from different sources to come up with a likelihood measure for the projection.

The fourth component is an oil spill model that is adopted mostly from the existing oceanographic and marine physics research. This module consists of four distinct submodels that models four different types of oil slick motion, that is, weathering, transport, dispersion and spreading (Paluszkiewicz, 1989).

The last component, scenario integration module, takes as the inputs a set of projections of oil slick movement with the likelihood measures, and produces a set of projections of the most likely scenarios of the oil slick. The functions it performs include merging those projections whose differences are insignificant, deleting those that are highly unlikely, and preparing for the output to be presented to users.

Finally, the system graphically presents a set of the most likely scenarios of oil slick movement with their corresponding likelihood measure to the user who can use this valuable information to help make the oil spill management decisions.

\section{KNOWLEDGE ACQUISITION (KA)}

Diverse types of knowledge are involved in the reasoning process in this system. Some knowledge is acquired during the off-line learning period while other are acquired at runtime. Some are "precise" like statistical measures and some are less precise like domain expert's intuition or preference. Here we refer to the off-line statistical information extraction as a kind of learning. In the rest of this section, we focus on the methods for acquiring different types of knowledge.

\subsection{Off-line knowledge acquisition}

Different kinds of statistical data are acquired off-line during the learning period. Fig. 2 depicts the different submodules that acquire statistical data from different sources. Data and knowledge flow in the off-line $\mathrm{KA}$ is indicated in dotted lines while the run-time KA in solid lines.

We derived three different kinds of statistical data from historical database of actual and forecast weather conditions. The cycle analysis submodule is responsible for deriving daily and seasonal patterns of weather conditions. The statistical data is represented in the form of conditional probabilities, such as the following expression for daily pattern $p_{i j}\left(W i n d(t) \in W_{j} \mid t \in H i\right)$

where $H_{i}$ represents the $i$ th hour of a day and $W_{j}$ represents the $j$ th wind (winds are 


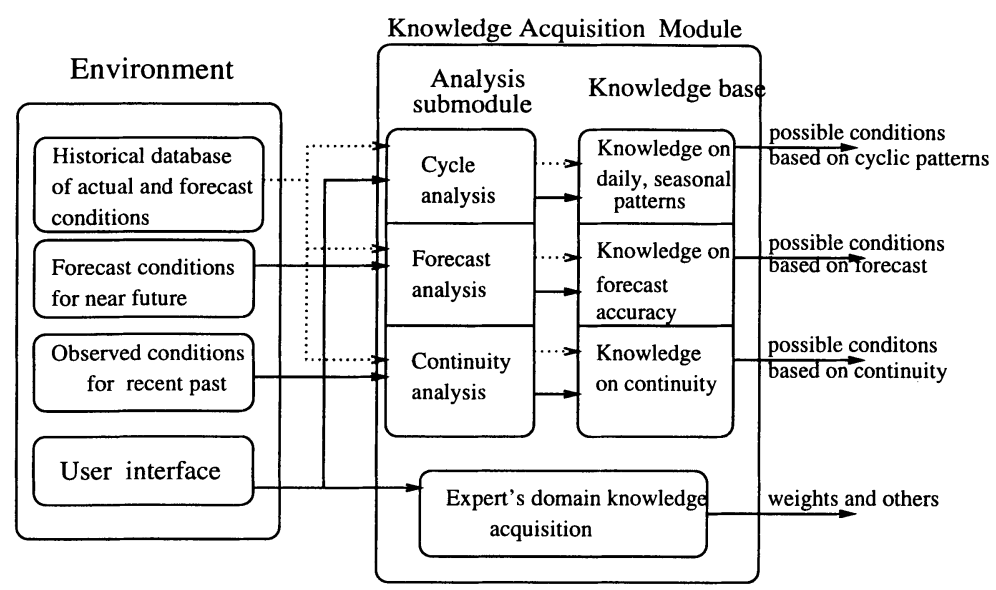

Figure 2 The Knowledge Acquisition Module

divided into $m$ groups). $p_{i j}$ is the conditional probability that the wind at $H_{i}$ hour is $W_{j}$. Similarly, we can derive the weekly and seasonal patterns. The probabilities are easily obtained using standard statistic techniques.

The submodules "forecast analysis" and "continuity analysis" are responsible for deriving the knowledge on the forecast accuracy and the the continuity from the historical data. In the historical database, we have data on the past forecast and observed weather conditions and the difference between the two is the forecast error. The degree of continuity between two winds $W_{i}$ and $W_{j}$ are represented by the probability of transition from $W_{i}$ to $W_{j}$. In a similar fashion, the knowledge on forecast accuracy and weather condition continuity is represented in probability tables.

These different kinds of statistical data are extracted off-line and stored in a knowledge base.

\subsection{Run-time knowledge acquisition}

In Fig. 2, the data and knowledge flow for the run-time knowledge acquisition is indicated by the solid lines. At run-time, when the current time and season are input into the knowledge acquisition module via a user interface, the cycle analysis submodule uses the daily, weekly or seasonal patterns that were built off-line to obtain a set of the most likely weather conditions based on the cyclic patterns. This is virtually a table look-up operation plus some constraint checking. In a similar manner, the forecast analysis and the continuity analysis submodules, using their respective statistical knowledge bases that have been built off-line, obtain a set of the most likely conditions based on their respective best knowledge. 


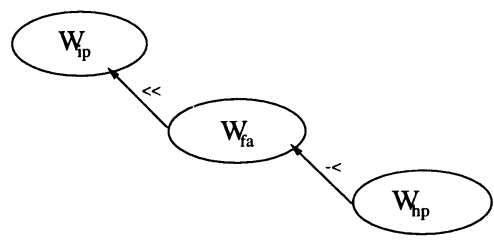

Figure 3 Influence Weight Graph for the Likelihood Measure of Weather Forecast

\subsection{Acquisition of expert's domain knowledge}

In Fig. 2, there is a link from the user interface to the submodule for acquiring expert's domain knowledge. The domain expert inputs his/her knowledge directly into the intelligent system via the user interface and the knowledge acquisition module extracts the domain knowledge and translates it into a form compatible with the statistical knowledge so that they can be processed together in the scenario generator module.

Heuristic knowledge such as domain expert's judgement and preference, and rules of thumbs like "near shore currents tend to run parallel to the shore" are often described in vague terms. For example, one of the major goals for this system is to generate a likelihood measure for a projection of oil slick movement. We identified three types of information which can influence the likelihood. They are the historical weather pattern for the season, the observed weather conditions of the recent past and the accuracy of the past weather forecast. However, the domain expert may favor one type of information over other, say, immediate past weather condition over historical weather pattern. The domain expert's preference and intuition are usually very difficult to quantify. We use a method described in (Weld, 1990) for extracting this type of imprecise knowledge. This method combines qualitative reasoning with fuzzy set theory (Zadeh, 1972) to acquire the influence weights. This method is based on the observation that domain experts usually hesitate to quantify the influence of variable $X$ on $Y$. However, it is relatively easy for the domain experts to express the relative weights of $X_{i}$ and $X_{j}$ on $Y$. The method attributes fuzzy value to weights from the linguistic description of the relative orders of magnitude.

We define $X_{1}, \ldots, X_{n}$ be a set of variables influencing the variable $Y$ with the respective weights $W_{1}, \ldots, W_{n}$. The linguistic description of orders of magnitude of the weights is a set of pairwise links between these weights and can be described by a formalism as shown below:

\begin{tabular}{ll} 
relation & verbal description \\
\hline$A<<B$ & A is much smaller than B \\
$A-<B$ & A is moderately smaller than B \\
$A==B$ & A is approximately equal to B
\end{tabular}

If we use a node to represent a weight and an edge to represent the fuzzy link (linguistic descriptor) between the two nodes $X_{i}$ and $X_{j}$, we have a directed acyclic graph as shown in Figure 3

In this example, the graph represents the following relations: $W_{f a}<<W_{i p}$ and $W_{h p}-<$ 
$W_{f a}$ where $W_{f a}, W_{i p}$ and $W_{h p}$ are the weights for the weather forecast accuracy, the immediate past weather condition and the historical weather pattern respectively. To compute the absolute value of the weights $W_{i}$, we assume that there exists a maximum weight $W_{\max }$ such that for any $W_{i}$, there exists a path from $W_{i}$ to $W_{\max }$. An algorithm to explore the weight graph from the top to the bottom in breadth-first fashion and to compute the intersect of the supports of each node's parent value along the way, will yield a set of numeric weights $\left(W_{1} \ldots W_{n}\right)$ in $[0,1]$.

\subsection{Integration of different types of knowledge}

The different types of knowledge are fused to produce a projection of oil slick movement and a likelihood measure of the projection. The fusion takes place in the scenario generator module.

The domain expert's knowledge on the relative weight of each type of information is fused into the belief network to produce a likelihood measure for a projection. Given $\left(W_{1}, \ldots, W_{n}\right)$ in $[0,1]$, we can normalize the weights and then use the resulting weights to weigh the conditional probabilities:

$\tilde{p}_{i}=p_{i} * \frac{W_{i}}{W_{\max }}$

where $\tilde{p}_{i}$ is weighted conditional probability and $W_{\max }=\max \left(W_{1}, \ldots, W_{n}\right)$

We use a Bayesian belief network to combine various weighted conditional probabilities and to compute the likelihood of the projection. A Bayesian belief network is a directed acyclic graph (DAG) whose nodes are the variables or propositions and whose arcs represent the existence of influence and are quantified by the conditional probabilities (Pearl, 1988). The weighted conditional probabilities for historical pattern, continuity and forecast for a state variable such as wind can be combined based on a belief network assuming the independence between the variables. Finally the likelihood measures for all state variables can be combined in a similar fashion to produce the final output of the likelihood measure for a given projection of oil slick movement.

\section{IMPLEMENTATION}

The intelligent system for predicting oil slick movement described in this paper is currently under development. Major issues in the implementation are discussed in this section.

First, there are various types of knowledges required in this system. A representation scheme that can modularize knowledge base by encapsulating knowledge of the same type in a relative independent unit is very desirable, since it would facilitate the system operation and maintenance. Secondly, a large amount of data is used to acquire different types of knowledge in this system. A method to provide uniform treatment of data and knowledge could facilitate the maintenance of the system, promotes the reuse of system functionalities and thus reduce the development cost.

An object-oriented paradigm is adopted to address the above issues and to implement the described system. We use an object-oriented representation for both data and high level knowledge. Related data and the knowledge are combined into a single unit, i.e., an 
object. Since data and other types of knowledge are treated uniformly, it simplifies the development process and reduces the development cost.

The user interface is to be developed using $\mathrm{X}$ window based utilities. The user is prompted to provide initial data value such as current wind velocity, wind direction, etc., via an application window. Then there are optional windows for the user to enter expert's domain knowledge such as the judgement on the reliability of historical weather data for this particular spill area, the geographical characteristics that affect the spill movement and others. If no domain expert's knowledge is entered, some default knowledge is assumed. The output of the system is in both statistical and graphical form. A table would list all inputs and the corresponding outputs. Various most likely scenarios for the oil slick movement are overlapped on the same plane and the intensity of color would represents the relative likelihood of a scenario.

A prototype system is being implemented in $\mathrm{C}++$ on Sparc workstation. Some realistic testing data has been collected for validate the system once the implementation is completed.

\section{SUMMARY}

The goal of this research is to build an intelligent system to predict the oil slick movement to support the decision making in the management of a marine oil spill. The problem is characterized by the high degree of uncertainty and impreciseness associated with the observational data and the operating environment. The key to produce a prediction that is useful to the decision maker in managing an on-going oil spill is to acquire and to incorporate into a prediction as much knowledge as possible to reduce the amount of uncertainty the decision maker has to face. The major contributions of this system are the integration of a knowledge base containing diverse types of knowledge with a set of mathematical models simulating various oil slick motions and the adoption of an object-oriented paradigm to address various implementation and knowledge representation related issues.

\section{REFERENCES}

J.W. Anderson. Characteristics of dispersion and water soluble extracts of crude and refined oils and their toxicity to estuarine crustaceans and fish. Marine Biology, 1974.

J.A. Fay. Physical processes in the spread of oil on a water surface. In Proceedings of the Joint Conference on the Prevention and Control of Oil Spills, pages 463-67. American Petroleum Institute: New York, 1971.

Theresa Paluszkiewicz and Charles F. Marshall. Comparison of techniques for forcing an oil spill trajectory model. In Proceedings of International Oil Spill Conference, pages 547-553, February 1989.

J. Pearl. Probabilistic Reasoning in Intelligent Systems: Networks of Plausible Inference. Morgan Kaufmann, 1988.

William B. Samuels, Norden E. Huang, and David E. Amstutz. An oil spill trajectory analysis model with a variable wind deflection angle. Ocean Engineering, 9(4):347-360, 1982. 
J. Tsao, J. Wolter, and H. Wang. Model-based understanding of uncertain observational data for oil spill tracking. 3rd Intl. Conf. on Industrial Fuzzy Control and Intelligent Systems, 149-154, 1993.

J. Tsao, J. Wolter, and H. Wang. Model-based explanation of uncertain observations. Proceedings of 1994 IEEE International Conference on Systems, Man and Cybernetics, 2578-2583, 1994.

S. Venkatesh. The oil spill behaviour model of the Canadian Atmospheric Environment Service. part I: Theory and model evaluation. ATMOSPHERE-OCEAN, 26(1):93-108, 1988.

S. Venkatesh. Model simulations of the drift and spread of the Exxon Valdez oil spill. ATMOSPHERE-OCEAN, 28(1):90-105, 1990.

D. Weld and J. De Kleer. Readings in Qualitative Reasoning about Physical Systems. Morgan Kaufmann, San Mateo, CA, 1990.

G. N. Williams, R. Hann, and W.P. James. Predicting the fate of oil in the marine environment. In Proceedings of the Joint Conference on the Prevention and Control of Oil Spills, pages 567-572. American Petroleum Institute: New York, 1974.

L. Zadeh. Outline of a new approach to the analysis of complex systems and decision processes. IEEE Trans. on Sys., Man and Cybernetics, 3, 1972. 